\title{
Multi Agent System Based Process Control in Wide Area Protection against Cascading Events
}

\author{
Zhou Liu, Zhen Chen \\ Dept. of Energy Technology \\ Aalborg University \\ Aalborg, Denmark \\ zli@et.aau.dk, zch@et.aau.dk
}

\author{
Haishun Sun \\ Huazhong University of Science and \\ Technology \\ Wuhan, China \\ Haishunsun@hust.edu.cn
}

\author{
Yanting $\mathrm{Hu}$ \\ School of Engineering Education \\ Glyndwr University \\ Wrexham, UK \\ y.hu@glyndwr.ac.uk
}

\begin{abstract}
In order to prevent long term voltage instability induced cascading events; a multi agent system (MAS) based wide area protection strategy is proposed in this paper. Due to some unexpected failures of control strategy execution or the consequent unexpected disturbance, the power system will face more complex emergent situation than planned. The process control strategy will be applied to improve the effectiveness and reliability of MAS based control strategy. The supervisory agent will help control center in the high level to manage not only the regular control process against the estimated emergent states, but also those unusual control process variations when unexpected situation is experienced. A hybrid simulation platform based on MATLAB/LabVIEW and real time digital simulator (RTDS) is set up to simulate a voltage collapse case in the power system of Eastern Denmark and demonstrate the effectiveness of the improved MAS based protection strategy.
\end{abstract}

Index Terms-Multi agent system, wide area protection, voltage instability, process control, hybrid simulation platform

\section{INTRODUCTION}

In N-1 (or N-k) post contingency stage, the issues of voltage instability and related cascading events have drawn a lot of attention in power system researches, when load demand increases drastically beyond the supply capability of generation and transmission system [1]. Many past power system blackouts were due to voltage instability induced cascading trips, such as the northeast U.S./Canada blackout in 1965, the western U.S. blackout in 1996, the Brazil blackout in 1999, and the southern Sweden and eastern Denmark blackout in 2003 [2,3]. It can be inferred that the emergent states, such as overload of transmission lines or generators, should be adjusted timely to avoid the protection relays tripping critical system components unexpectedly.

Until now, many previous research efforts have been made to develop special system protection schemes (SPS) based on wide area measurement system (WAMS) against voltage instability and subsequent cascading trips [3]-[8]. Most of them focus on analyzing the influence factors of cascading events and developing the countermeasures for specific one or two factors. The process control of integrated countermeasures against the complex situations in cascading events is less considered. It is very important for the operation reliability of control strategy, if the only one or two countermeasures fail to prevent the cascading events.

In this paper, a multi agent based wide area protection system is proposed to prevent the cascading events due to voltage instability. The distributed relays and controller will work as device agents in an intelligent environment which is formed by multi agent system. Besides the traditional function can be operated automatically, the distributed agents can be programmed to fulfill the extra function according to external requirements. In this way, these distributed agents are organized by the control center (CC) of MAS to achieve the global aim of preventing wide area disturbance.

According to different emergent states detected by the relay agents, different control methods will be defined correspondingly and executed by control agents, such as adaptive relay setting, load shedding, smart islanding, load tap changer control and etc. When the system experiences cascaded blackout, the single control method may be not enough to prevent the diverse emergent states in a long run, especially when some unexpected failures of control strategy execution or some unexpected disturbance occur [9]. In order to promote the reliability of this wide area protection scheme, the hierarchical control structure and process control strategy is adopted and conducted by related control agents for dealing with the complex situation when some control strategy fails to prevent the related emergent state.

A study case of voltage instability induced cascading events will be simulated on the power system model of eastern Denmark. A hybrid simulation platform built with RTDS and MATLAB/LabVIEW is established to demonstrate the effectiveness of the proposed protection strategy. In the rest of the paper, the basic control process of proposed protection strategy will be presented in Section II; the MAS based process control will be described in Section III; in Section IV, validation of the strategy will be demonstrated with a voltage instability case in the hybrid simulation platform; and finally, the conclusion will be made in Section V. 


\section{The BASIC Process OF PROTECTION STRATEGY}

\section{A. Basic Structue of Multi Agent System}

The basic structure of MAS proposed in [10] is adopted in this paper, as shown in Figure 1.

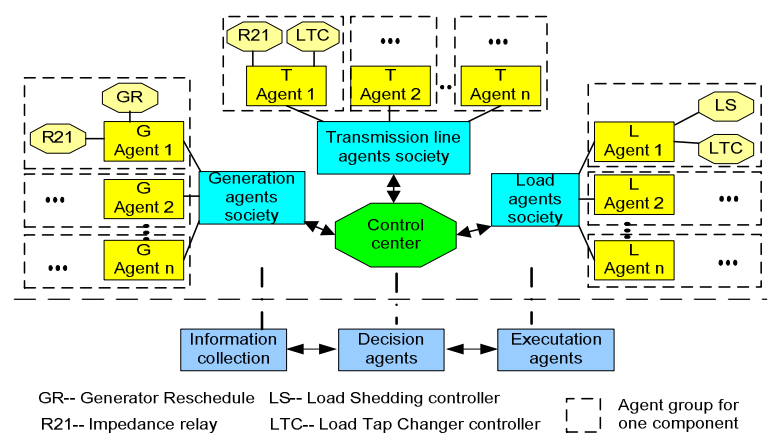

Figure 1. The basic structure of MAS based protection system

This MAS based system protection scheme adopts a threelevel hierarchical control structure, which is comprised of distributed agent level, cooperation society level and central processing level. Meanwhile, every agent in MAS has three basic functions: information collection, decision making according to the prevailing state, and decision execution.

\section{B. Basic Task Flow of Protection and Control Strategy}

With the aim of adjusting the power system emergent states timely before the unexpected relay trips, the basic task flow process can be design according to [11], as shown in Figure 2, where the black arrows represent the data flows and the blue arrows represent the task flows.

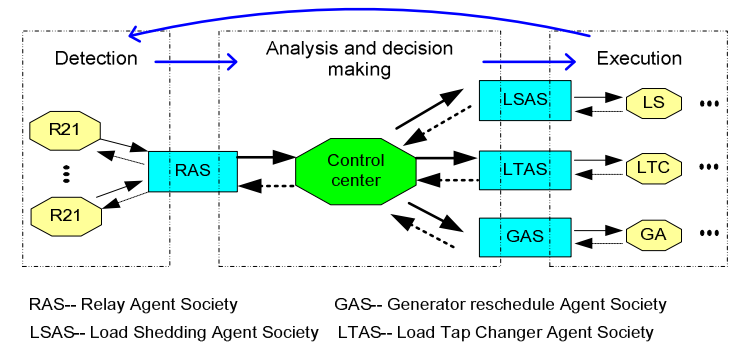

Figure 2. The process of basic task flow

Firstly, the overload states of power system components are detected and identified by distributed relays. Then through the cooperation and communication level, the identified emergent states of system are analyzed by $\mathrm{CC}$, and the related control strategies (including load tap changer control, load shedding and generator reschedule) are produced based on sensitivity method. Finally, the distributed controllers will execute the defined control strategies immediately when they receive the orders from $\mathrm{CC}$. The detection will check if the emergent state has been adjusted successfully and prepare for a new round.

\section{1) Detection of Emergent States}

In order to detect the voltage stability associated overload emergent states, the method proposed in [12] is discussed here further, which can be depicted in Figure 3. Making the generator back up relay as an example, at the power factor angle $\varphi_{i}, Z_{a i}$ and $Z_{G s e t i}$ are the measured impedance and setting value of the backup relay. The impedance plane in the first quadrant can be divided into four areas, which are labeled as Normal area, Urgent area, Zone 3 area and Zone 12 area respectively. These four areas correspond to four states of this generator, which are Normal state, Urgent state, Zone 3 start state and Tripping state. Zone 3 area is the operation area of zone 3 element in well-know three-zone impedance relays. Similarly, Zone 12 area represents the operation areas of zone 1 and zone 2 elements. Normal area represents the normal state that no capability limits of the generator are reached, and the area between Normal area and Zone 3 area is named Urgent area where the generator reaches its capability limits.

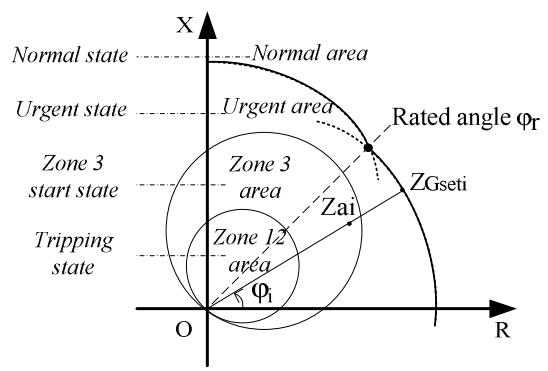

Figure 3. Areas for operation states of a impedance relay on generator $i$

As for an impedance relay on transmission line, the impedance plane can be similarly divided into three areas, i.e. zone 12 area, zone 3 area and normal area. The setting value of zone 3 relay is defined according to relay loadability limit [11]. In this way, the overload situation of each component in the transmission network can be detected in real time by distributed relays.

\section{2) Solutions for Identified Emergent states}

When the $\mathrm{CC}$ receives the signals and information through the cooperation level, based on sensitivity analysis, the diverse solutions for different emergent states can be made directly and quickly [12], which is shown in Figure 4.

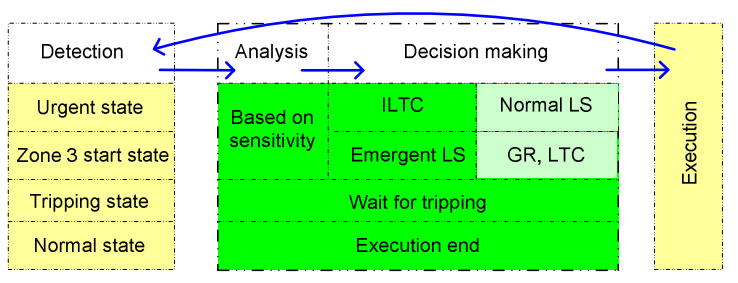

Figure 4. Solutions for identified states

When the urgent state has been detected, the inverse load tap changer control (ILTC) will be adopted as main countermeasure to adjust system state, meanwhile the normal load shedding (LS) with 10 s delay will be used as compensation for local load balance. When Zone 3 start state has been detected, the breaker will be tripped in about $500 \mathrm{~ms}$ normally, so the emergent LS will be chosen as main counter measure in order to adjust this more emergent state timely before this unexpected trip, while the Generator reschedule and tap changer control will be used as compensation. In Tripping state, the $\mathrm{CC}$ will do nothing but waiting for the 
traditional function of the critical relay to cope with it. And the normal state will be regarded as a secure operation state or a return state after the execution of control strategy.

\section{Multi AgEnt System BASEd Process CONTROL}

Based on the previous contents, it can be inferred that whether the emergent states can be adjusted and prevented successfully mainly depends on the fast and effective communication and execution. If the related countermeasures fail to prevent the emergent states and subsequent trips, the system will experience a more complex situation, even the cascading trips. So in order to improve the effectiveness and reliability of this protection strategy, these unexpected and complex situations should be carefully considered.

\section{A. Advanced structure of MAS for process control}

In order to deal with complex situation, an improved MAS based hierarchical control system is proposed here, as shown in Figure 5. The agent named Supervisory control center (SCC) is responsible for general supervision of the control process performance. It provides supervisory compensation control against process fluctuations, such as normal control strategy fail to execute. The knowledge of this agent depends on global shared variables of the objects and operator experience. Also, human-machine interface (HMI) can be built on remote computer to support the supervisory controls by operators.

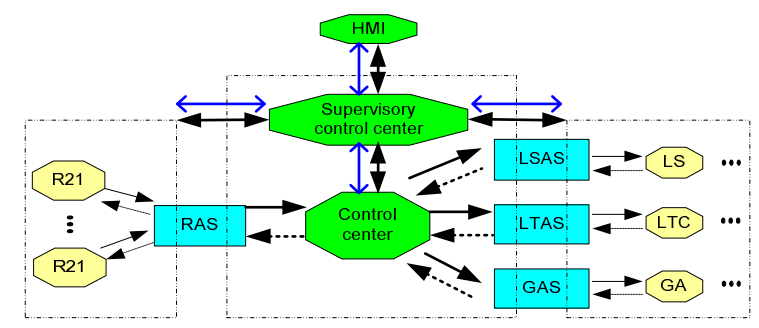

Figure 5. Multi agent based Process control

\section{B. Global shared variables and new solutions}

The states of relays and controllers will be measured and defined as shared variables which the state supervisor in SCC can detect. Also, the state transitions of control process will be traced and defined as global shared variables. In this way, the supervisory control center can easily identify the variations of control process. Then the operation supervisor can make process regulation against the identified abnormal process variation. The basic functions and task flow is shown in Figure 6.

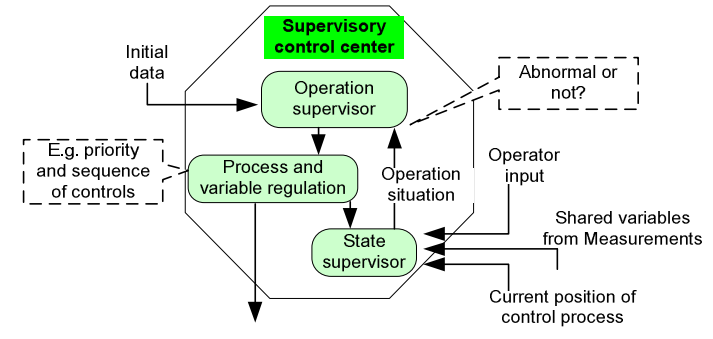

Figure 6. The structure of supervisory control center
For example, in the case of countermeasure execution failure, the supervisory agent will change the priority level of existing countermeasures. If the zone 3 start state has been detected, the emergent LS, which is regarded as the main solution in former control settings to prevent the cascading events, fails to be executed; then the smart islanding will be a good alternative to save some part of system timely. Then the SCC will define the smart islanding as the countermeasure with highest priority, which will be initiated immediately when the emergent LS fails and related unexpected trip occurs; meanwhile, the emergent LS will be defined as compensation method to be initiated secondly against the power unbalance in the separated islands. The algorithm of LS and smart islanding are based on online sensitivity analysis and graph theory, which will be not discussed here. The process regulation can be depicted in Figure 7.

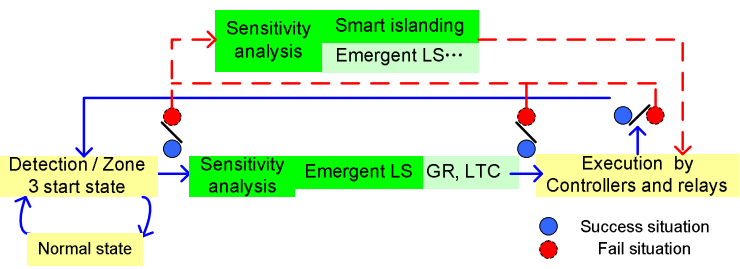

Figure 7. Process tracing and regulation

\section{Hybrid simulation platform}

In this paper, a hybrid simulation platform is built to implement the proposed protection strategy, as shown in Figure 8. By using the standard models in the library of RTDS, the simplified power system model of Eastern Denmark is developed together with the distributed relays and controllers, and the task of information exchange between different agents and societies is conducted [13]. Most functions in $\mathrm{CC} / \mathrm{SCC}$ and the sensitivity based control algorithm [11] are implemented in MATLAB/LabVIEW. The global shared variables are shared between distributed operation systems through the shared variable engine which is based on NI Publish-Subscribe Protocol (NI-PSP) data transfer protocol [14]. The CC and SCC are built in real time operation system (RTOS) with LabVIEW, which implement the real time control strategy together with RTDS.

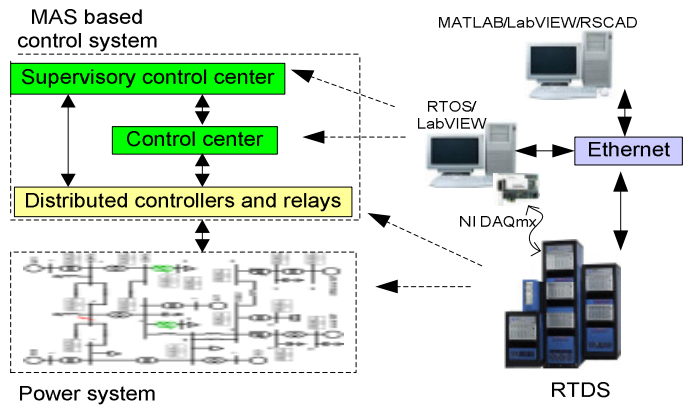

Figure 8. MAS based control system implementation

\section{CASE STUdy}

In this paper, the simplified power grid of eastern Denmark is adopted as test system, which is built in RSCAD/RTDS with two racks, as depicted in Runtime/RTDS 
and shown in Figure 9. In this system, there are 19 buses from $0.7 \mathrm{kV}$ to $400 \mathrm{kV}$, four central power plants and their control (G01, G11, G21, G22), a Static VAR compensator (SVC), several consumption centers, a lumped equivalent of local wind turbines and an equivalent of a large offshore wide farm [15]. The four central power plants have OEL model [18]. The generators' capability curves are modeled in R-X plane as backup relay settings, which are used to initiate alarms to CC. These settings apply $I_{f d 01 \max }=I_{f d 11 \max }=I_{f d 21 \max }=2.68 \mathrm{pu}$, $\mathrm{I}_{\mathrm{fd} 22 \max }=2.85 \mathrm{pu}$ and $\mathrm{V}_{\min }=0.9 \mathrm{pu}$. The exponential restoration load model [2] is applied for consumption center Load 1 and Load 2. And Load 3 and Load 4 are modeled by constant power load. The LTCs are modeled on transformer T8, T10, T13 and T14. The detailed parameters can be found in [15].

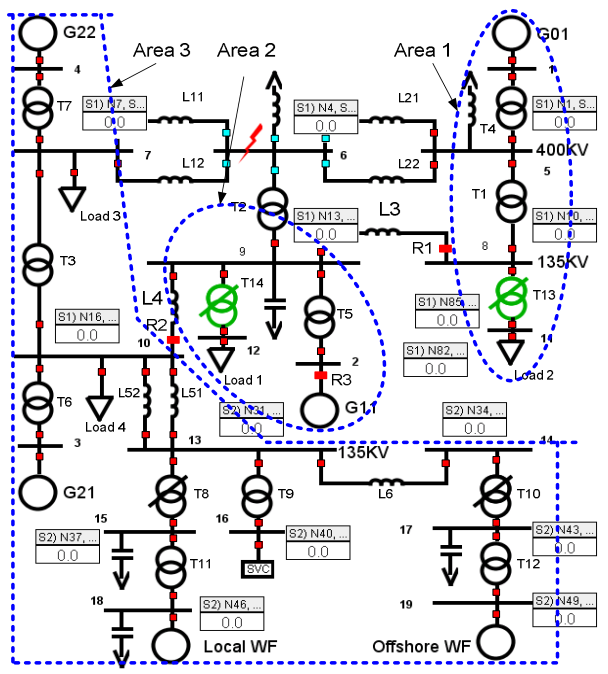

Figure 9. The structure of supervisory control center

\section{A. The scenarios of voltage collapse}

In order to verify the MAS based protection strategy, a severe case of voltage instability is initiated by a three phase short circuit fault applied on bus 6 at about 11s, as shown in Figure 9. The relays trip all the lines and transformers connected to bus 6 within $0.1 \mathrm{~s}$. The red cubes represent the breakers are on, and the blue ones are off. Then, this system survives after an oscillation of about 24s. Due to load restoration by the LTC and load dynamics, the stressed network cannot support the increasing load. The voltage collapse progress can be observed in two situations with the traditional relay blocked or not, as shown in Figure 10.

In Figure 10 (a), when the relays are blocked in the post fault stage, voltage collapse still occurs at about 210 s due to all the generators hit the capability limits. In Figure 10 (b), the backup relay R1 on line L3 is triggered at about 87s to trip this line at $88 \mathrm{~s}$ unexpectedly. Then the part Area 1 in Figure 8 is separated from the whole system and survives. Thereafter, a large amount of power flow transferred from Area 3 to Area 2 through L4 to meet the increasing demand in Load 1, L4 is thus heavily overloaded and tripped by the backup relay R2 at about 93s, then the remaining network splits into Area 3 and Area 2. Due to the big unbalance between the generation and consumption in Area 3 and Area 2, frequency and voltage collapse together in both areas.

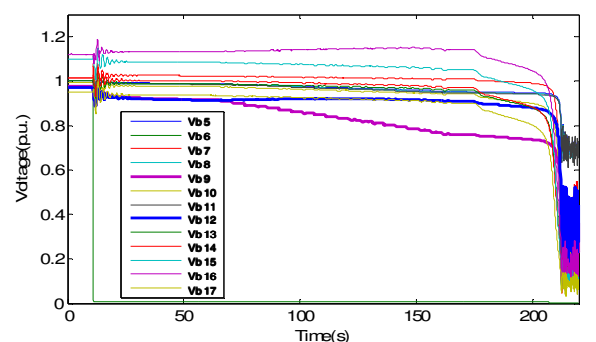

(a) Collapse with relays blocked in the post fault state

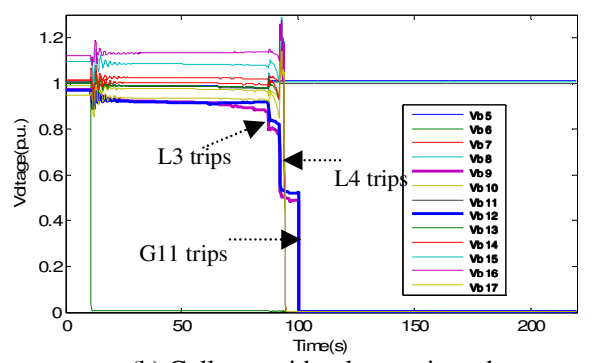

(b) Collapse with relays activated

Figure 10. Voltage collapse progress in the post fault stage

\section{B. Protection strategy with normal control process}

Based on normal control process described in section II, the distributed controller agents will receive the emergent orders and execute them immediately. It can be seen from Fig. 11 that after timely LS execution at $87.5 \mathrm{~s}$, the bus voltages are recovered to a safe level over 0.9pu. Also in Fig. 12, the critical relays are reset back when measured impedance in red color moves into Normal area (the black loci represent the collapse situation with relays being blocked, and the blue loci represent the collapse situation with relays activated).

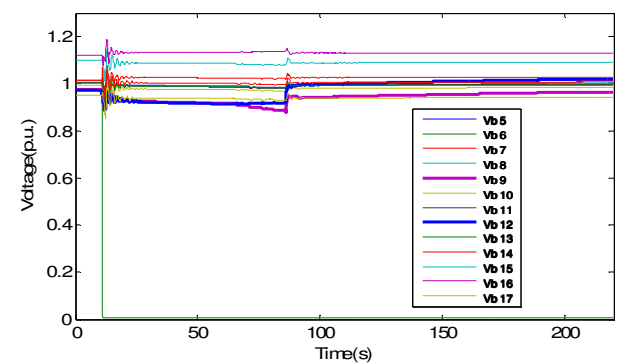

Figure 11. Bus voltages with protection strategy executed successfully
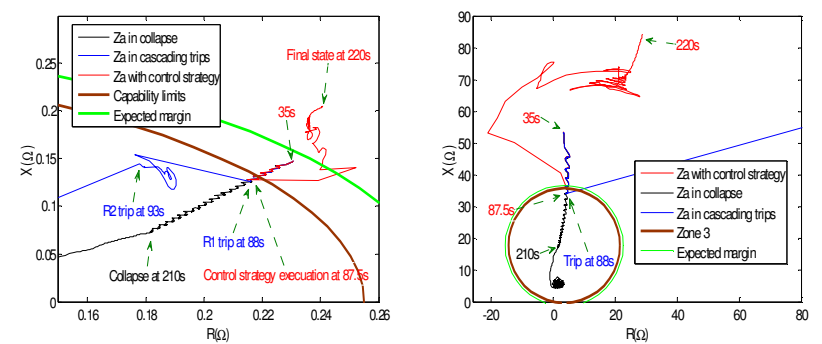

(a) Impedance loci of relay R3 on G11 (b) Impedance loci of relay R1 on L3

Figure 12. Impedance loci of relays in LS susccessful situation 
According to sensitivity analysis, the control points and control amounts can be easily obtained, as shown in Table I.

TABLE I. EMERGENT LS AND GR STRATEGY

\begin{tabular}{|c|c|}
\hline For Load 1 and G11 & For Load 2 and G01 \\
\hline 518.4 MVA & 7.12 MVA \\
\hline
\end{tabular}

However, if the LS controllers fail to execute the orders from $\mathrm{CC}$, then the zone 3 start state cannot be adjusted timely, and the unexpected cascading trips will occur inevitably. The process of this collapse is similar to Figure 10 (b).

\section{Protection strategy with advanced process control}

In order to deal with the situations of execution failures, the proposed advanced MAS based process control strategy is taken into effect to monitor the whole process of protection scheme and regulate the abnormal process timely to survive the parts of power system.

In this case, if the LS controller on Load 1 fails to shed the load, the R1 will trip the L3 within a preset delay. Then the SCC will register this execution failure and the R1 trip which will be identified as an abnormal process of control strategy. The regulation orders will be sent from SCC to CC, which will interrupt the current task and initiate a new task, e.g. smart islanding in this case, to change the original control process and further adjust the deteriorated emergent state of power system. The front panel of control system built in LabVIEW can be seen in Figure 13.

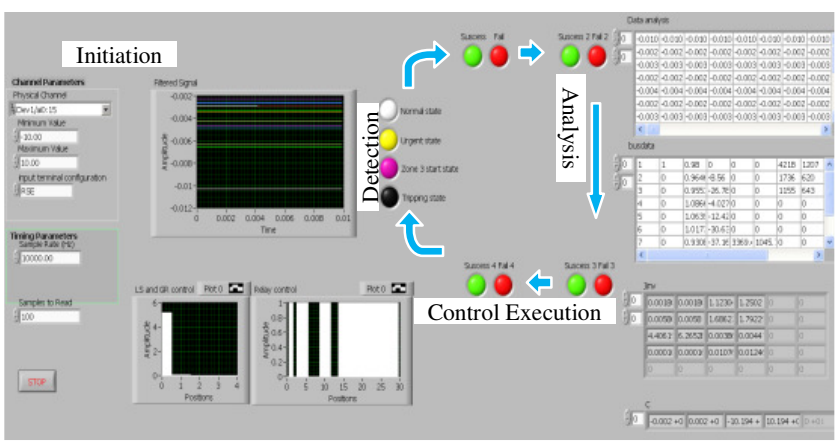

Figure 13. The front panel of control systemin LabVIEW

With smart islanding (SL) strategy initiated, the L4 will be tripped by $\mathrm{R} 2$ immediately when $\mathrm{R} 2$ received the orders from CC. Then the system will be actively separated into three areas, as shown in Figure 9. At the same time, the related LS and GR will be initiated to keep the power balance in these three islands. After new tasks and process have been executed successfully, the bus voltages in islands are recovered to a safe level beyond $0.9 \mathrm{pu}$, as shown in Figure 14. Also, the critical relay R3 on G11 is reset back to Normal state when measured impedance in red color moves into Normal area, as shown in Figure 15.

In these areas, about $55 \%$ of system loads has been saved together with $74.1 \%$ of system generation. Total loss amount of load in Area 2 is $1515.78 \mathrm{MVA}$, the total loss of generator capability in Area 3 is 1710 MVA and the wind farms have been shut down. But compared to total blackout, parts of system survived under the new process control strategy when the normal one failed.

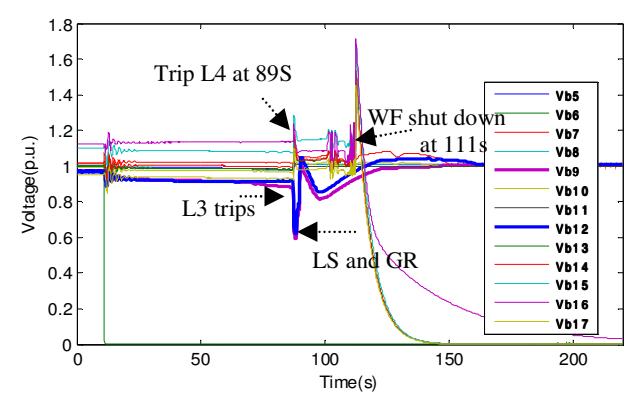

Figure 14. Bus voltages with SL, LS and GR

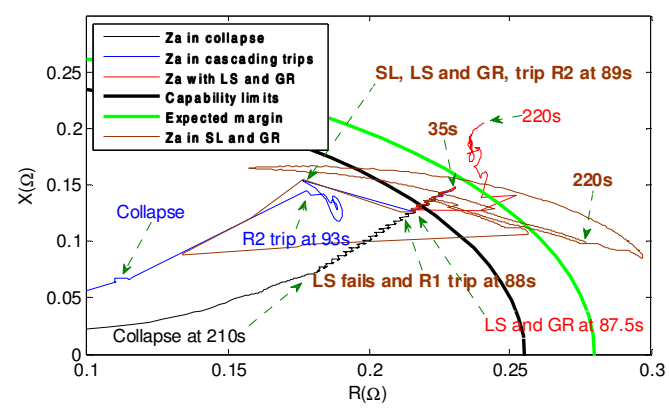

Figure 15. Impedance loci of relay R3 on G11

\section{CONCLUSION}

In this paper, a MAS based wide area protection strategy is proposed and discussed in order to prevent post fault voltage instability induced cascading events. Moreover, in order to improve the effectiveness of this wide area protection scheme, a hierarchical process control strategy is proposed for dealing with the abnormal situations when normal control strategy fails to prevent the related emergent state. A supervisory control center is simulated to monitor the normal control process in control center and provide timely regulation against abnormal processes. A hybrid simulation platform built in RTDS and MATLAB/LabVIEW is used to implement and verify the proposed protection strategy. It has been demonstrated that the proposed protection strategy with process control is more reliable on preventing the severe voltage instability case.

\section{REFERENCES}

[1] C. Taylor, Power System Voltage Stability, McGraw Hill Inc., 2004

[2] T. Cutsem and C. Vournas, Voltage stability of Electric Power System, Springer, 2008.

[3] S. Corsi, "Wide area voltage protection", IET Generation, Transmission \& Distribution, vol. 4, pp. 1164 - 1179, 2010.

[4] J. McCalley, O. Oluwaseyi, V. Krishnam, R. Dai, C. Singh, K. Jiang, "System Protection Schemes: Limitations, Risks, and Management," Final Report from PSERC, 2010.

[5] H. Song, B. Lee and V. Ajjarapu, "Control strategies against voltage collapse considering undesired relay operations", IET Generation, Transmission \& Distribution, vol. 3, no. 2, pp. 164-172, 2009.

[6] S. Li, N. Yorino, M. Ding, Y. Zoda, "Sensitivity Analysis to Operation Margin of Zone 3 Impedance Relays with Bus Power and Shunt Susceptance", IEEE Transactions on Power Delivery, vol. 23, no. 1, January 2008.

[7] M. Jonsson, "Protection Strategies to Mitigate Major Power System Breakdowns", PhD thesis, Goteborg, Sweden, 2003. 
[8] R. Diao, K. Sun, V. Vittal, R. Keefe, R. Michael, S. Dwayne, S. Sanjoy, "Decision Tree-Based Online Voltage Security Assessmen Using PMU Measurements", IEEE Transactions on Power Systems, vol. 24, no.2, May, 2009.

[9] Z. Liu, Z. Chen, H. Sun, C. Liu, "Control and Protection Cooperation Strategy for Voltage Instability”, 47th International Universities' Power Engineering Conference (UPEC), 2012.

[10] Z. Liu, Z. Chen, H. Sun, C. Liu, "Emergent Load Shedding Strategy Based on Sensitivity Analysis of Relay Operation Margin against Cascading Events", IEEE PES International Conference on Power Systems Technology (Powercon), 2012.
[11] Z. Liu, Z. Chen, H. Sun, C. Liu, Y. Hu, "Multi Agent System Based Wide Area Protection against Cascading events", The 10th International Power and Energy Conference (IPEC), 2012.

[12] V. Tan, D. Yoo, J. Shin, M. Yi. "A Multi Agent System for Hierarchical Control and Monitoring", Journal of Universal Computer Science, 2009.

[13] RTDS Technologies Inc. http://www.rtds.com

[14] National Instruments Inc. http://www.ni.com

[15] V. Akhmatov, "Analysis of dynamic behaviour of electric power systems with large amount of wind power", Ph.D. dissertation, Dept Electric Power. Eng., Technical Univ. Denmark, Kgs. Lyngby, 2003. 